Methods: Patients with PsA over the age of 18-year-old who met the CASPAR classification criteria were underwent clinical and laboratory evaluation by using standardized protocol and case report form, from two universities outpatient's clinics. PtGA and PhGA, VAS-pain, fatigue, BASDAI, BASFI, health-related QoL were recorded at baseline visit. Pearson correlation coefficient were calculated for PtGA and PhGA. Linear regression model were used to asses relationship between global assessments and clinical finding.

Results: A total 144 patients with PsA (male 51, female 93) were included. Symptom duration (7.8 \pm 7.5 vs $11.3 \pm 11.2)$, VAS-pain (4.4 \pm 2.6 vs $4.9 \pm 2.6)$, PtGA $(4.7 \pm 2.5$ vs $5.0 \pm 2.4)$ and PhGA $(4.0 \pm 2.2$ vs $4.4 \pm 2.2)$ were similar between male and female patients with PSA ( $p>0.05)$. VAS-fatigue was higher in female patients with PsA than male ones $(5.9 \pm 2.7$ vs $4.4 \pm 2.8, p=0.001)$. Disease activity measurements such as, tender and swollen joint count, DAS28, BASDAI and acute phase reactant were similar for both sex. Correlation coefficient of Physician's global assessment was higher than PtGA in various clinical findings. The highest correlation coefficient was observed in VAS-pain $(r=0.784)$ and followed by SF36-bodily pain $(r=-0.613)$, BASDAI $(r=0.587)$, NHP-physical activity $(r=0.584)$, VAS-fatigue $(r=0.555)$, BASFI question $5(r=0.551)$ for PhGA $(p<0.0001)$. However the highest correlation coefficient was observed in VAS-pain $(r=0.690)$ and followed by NHP-physical activity $(r=0.536)$, BASFI question 10 $(r=0.512)$, BASDAI $(r=0.499)$, SF36 $(r=-0.490)$ and BASFI total score $(r=0.484)$ for PtGA $(p<0.0001)$. Statistically significant positive linear relation was observed between global assessment and clinical parameters (VAS-pain, fatigue, BASDAI, BASFI, DAS28, NHP-physical activity) by using simple linear regression model. VAS-pain and BASDAI question 1 explained $83.3 \%$ of PtGA as a predictor in multiple linear regression model. However, VAS-pain and DAS28 explained $68.4 \%$ of PhGA as a predictor in multiple linear regression model (table 1).

Table 1. Results of multiple linear regression of PtGA and PhGA

\begin{tabular}{|c|c|c|c|c|c|}
\hline & $\mathrm{R}^{2}$ & B & SE & $t$ & $\mathrm{p}$ \\
\hline \multicolumn{6}{|c|}{ Patient's global assessment } \\
\hline Constant & 0.833 & 0.597 & 0.311 & 1.920 & 0.060 \\
\hline VAS-pain & & 0.665 & 0.061 & 10.939 & $? 0.0001$ \\
\hline \multicolumn{6}{|c|}{$\begin{array}{l}\text { BASDAI-1 How would you describe the overall level } \\
\text { of fatigue/tiredness you have experienced in the }\end{array}$} \\
\hline past week? & & 0.205 & 0.063 & 3.243 & 0.002 \\
\hline \multicolumn{6}{|c|}{ Physician's global assessment } \\
\hline Constant & 0.684 & 0.399 & 0.310 & 1.285 & 0.201 \\
\hline VAS-pain & & 0.587 & 0.050 & 11.846 & $? 0.0001$ \\
\hline DAS 28 & & 0.332 & 0.096 & 3.453 & 0.001 \\
\hline
\end{tabular}

Conclusions: Assessment of disease activity as well as clinical parameters is considered to be complex in PsA. Patient's and physician's have different perspective of disease activity. Severity of pain and fatigue may be predictor of PtGA whereas; pain and objective disease activity measurement may be predictor of PhGA in patient with PsA.

Disclosure of Interest: None declared

DOI: 10.1136/annrheumdis-2017-eular.5396

\section{AB0752 CONSENSUS STATEMENT ON THE USE OF METHOTREXATE IN PSORIATIC ARTHRITIS PATIENTS}

J. Tornero ${ }^{1}$, E. Loza ${ }^{2}$, J.D.D. Cañete ${ }^{3} .{ }^{1}$ Rheumatology, Hospital de Guadalajara, Guadalajara; ${ }^{2}$ Instituto de Salud Musculoesquelética, InMusc, Madrid;

${ }^{3}$ Rheumatology, Hospital Clínic e IDIBAPS, Barcelona, Spain

Background: Methotrexate (MTX) is the cornerstone in rheumatic diseases, including psoriatic arthritis (PsA), but the complexity of PsA and the paucity of randomised controlled studies and strategic trials need a clear analysis.

Objectives: To develop recommendations for the management of MTX in PsA (musculoskeletal manifestations) based on best evidence and experience

Methods: The coordinators formulated 14 questions about MTX use (indications, efficacy, safety) in patients with PsA. A systematic literature review was performed to answer these questions. Using this information, inclusion and exclusion criteria were stablished as well as the search strategies (Medline, Embase and the Cochrane Library), that were designed by an expert librarian. Two different reviewers selected the articles, first by title and abstract, then by detailed review, and collected data independently. Evidence tables were produced. With this evidence the coordinators proposed 12 preliminary recommendations that will be discussed and voted in a nominal group of expert meeting. The level of evidence and grade of recommendation will be defined using the Oxford Center for Evidence Based Medicine. Agreement will be stablished if at least $80 \%$ of the experts voted yes (yes/no).

Results: A total of 12 preliminary recommendations on the use of MTX in PsA were proposed (see table).

Conclusions: This document aims to help to answer usual clinical questions and facilitate decision making when treating PsA patients with MTX.

Disclosure of Interest: J. Tornero: None declared, E. Loza Grant/research support from: Abbie, Pfizer, Roche, Gebro, BMS, UCB, J. Cañete: None declared DOI: 10.1136/annrheumdis-2017-eular.1395
Abstract AB0752 - Table 1

\# Recommendation LE; GR

In patients with active peripheral disease (oligo-polyarthritis), the use of a $1 \mathrm{~b} ; \mathrm{A}$ csDMARD is recommended, MTX preferred, principally in cases of relevant skin involvement

2 A starting dose of at least $10-15 \mathrm{mg} / \mathrm{w}$ is recommended, taking into account disease severity and patients prognostic factors

3 In case of insufficient response to MTX starting dose, a quick dose increase should be performed up to $25-30 \mathrm{mg} / \mathrm{w}$ in approximately $8 \mathrm{w}$

4 Oral MTX is recommended, but parenteral MTX should be considered in patients $5 ; \mathrm{C}$ with high disease activity, those needing $>15 \mathrm{mg}$ oral/week, in those with low adherence to oral MTX, polypharmacy, obese patients, in order to avoid dosing errors, and always taking into account patients preferences

5 When the treatment target is reached for at least 6 months, then a dose adjustment is recommended in order to maintain the treatment target with the minimal MTX dose

6 Despite of lack of well designed clinical trials to demonstrate the efficacy of MTX in PsA, data coming from clinical trials using MTX as placebo, treat to target studies and years of rheumatologist accumulated clinical experience, all are in favor of MTX efficacy in peripheral PsA

7 In patients with peripheral PsA and enthesitis/dactylitis, MTX could be an option 2a; B along with steroids injections (if needed)

8 In general, combined therapy with MTX and other csDMARD is not recommended 2a; B

9 If a biologic therapy is indicated, MTX continuation should be individually $1 \mathrm{~b} ; \mathrm{A}$ evaluated in each patient

10 MTX improves anti-TNF $\alpha$ survival (monoclonal antibodies, mainly chimeric antibodies)

11 No special safety problems, regarding to rheumatoid arthritis patients, have been $1 \mathrm{~b} ; \mathrm{A}$ reported with MTX in PsA patients

12 PsA is a chronic disease in which MTX discontinuation is not recommended if $\quad 3 a ; C$ there is maintained efficacy and the drug is well tolerated

\section{AB0753 ARTERIAL STIFFNESS INCREASE IN THE EARLY PHASE OF ARTHROPATHY RELATED WITH PSORIATIC ARTHRITIS IS NOT FURTHER MODIFIED BY STABLE PROLONGED RETENTION OF A MINIMAL DISEASE ACTIVITY CONDITION}

F. Fischetti, F. Bozzao, M. Rovina, G. Simon, M. Bardelli, R. Carretta. University of Trieste Italy, Trieste, Italy

Background: Increased large arteries wall stiffness (AS) is a well known independent morbidity and mortality cardiovascular risk factor, not only related to hypertension and diabetets, but also induced by long standing systemic inflammation, as observed in inflammatory rheumatic or gut chronic diseases. Psoriatic arthritis (PsA) was also shown to increase cadiovascular morbidity, but this observation was commonly related with the associated occurrence of long term arthritis, disease activity and CV traditional risk factors

Objectives: To assess whether in a selected group of PsA patients, affected by recent onset (4-12 months) arthritis, not suffering from CV disease risk factors, evidence could be obtained of an early alteration of arterial wall function as expressed by an increased arterial pulse wave velocity (aPWV) and secondary reduced tonometric subendocardial viability ratio (tSEVR).

Further, we evaluated if a 18 months (Mth) treatment with synthetic disease modifying antirheumatic drugs (sDMARD) other than cyclosporine, leading to a demonstrable target of clinical "minimal disease activity"(MDA) could modify such possible modifications

Methods: Conclusive data were obtained in a selected goup of 12 PsA patients (Pts) (CASPAR criteria classified) (M/F, 6/5; mean age, 50.8; range, 40-65) not suffering from axial spondylitis who firsly underwent (without any previous treatment) aPWV measurement (PulsePen, Diatecne Srl, Milan) and tSEVR calculation, and then were re-evaluated for the same calculations after a 18 Mth time of sDMARD treatment. Each of the PsA Pts had a 3-4 Mth follow up and showed to reach the target of stable MDA (with also ultrasound confirmed remission of active synovitis) after a 4-8 Mth treatment. Before treatment, the group of PsA Pts was compared with an age, body weight, CV parameters and risk factors-matched control group of voluntary 22 healthy subjects (M/F, 11/11; mean age, 51.3; range, 41-67)

Results: Before any treatment, aPWV was higher in the group of patients with PsA than in control subjects (median, $8.667 \mathrm{~m} / \mathrm{s}$ vs $6.963 \mathrm{~m} / \mathrm{s}, \mathrm{p}<0.02$ ) while tSEVR was decreased (median, 1.44 vs $1.50, p<0.05$ ). Aortic PWV was not modified after the sDMARD treatment $($ median $\mathrm{m} / \mathrm{s}$, before $=8.691$, after $=8.048$, $p=0.55)$, despite statistically significant improvements of the disease activity scores (DAS28; modified CPDAI; DAPSA) as well as cutaneous PASI, and stable retention of a MDA condition. A direct correlation (Spearman rank) between aPWV and DAS28 (rho=0,70; p-value =0,04), BASDAI (rho=0,77; $p$-value $=0,01$ ), and $\mathrm{HAQ}$ (rho=0,66; $p$-value=0,05) was found, not instead between aPWV and ESR or CRP. Pts with PSA, at the end of the follow up, had increased levels of systolic $(134,1 \pm 14,3$ vs $122 \pm 11,2, p<0,05)$ and diastolic $(82 \pm 7,4$ vs $73,5 \pm 6,6$, $\mathrm{p}<0,05)$ blood pressure, with unmodified heart rate

Conclusions: Early onset of PsA seems to be associated with already established and stable increase of AS. Subclinical previous phase of inflammation and length of psoriatic disease could be addressed as possible causes

Disclosure of Interest: None declared

DOI: 10.1136/annrheumdis-2017-eular.5528 